\title{
A correlation of hysterolaparoscopic findings and BACTEC culture in infertility
}

\author{
Mahesweta Guru, Prasad Yeshwant Deshmukh*
}

Department of Obstetrics and Gynecology, LTMMC and GH, Sion, Mumbai, India

Received: 21 January 2017

Revised: 06 March 2017

Accepted: 07 March 2017

\author{
*Correspondence: \\ Dr. Prasad Yeshwant Deshmukh, \\ E-mail: drpydeshmukh@gmail.com
}

Copyright: (C) the author(s), publisher and licensee Medip Academy. This is an open-access article distributed under the terms of the Creative Commons Attribution Non-Commercial License, which permits unrestricted non-commercial use, distribution, and reproduction in any medium, provided the original work is properly cited.

\section{ABSTRACT}

Background: Infertility is agonising condition. Tuberculosis is an important health problem worldwide. One third of the world's population is currently affected with tuberculosis. Hysterolaparoscopy is a well-recognized procedure for the diagnosis of infertility. Culture for TB bacilli is the gold standard for diagnosis of genital TB.

Methods: Infertile women undergoing dilatation and curettage with hysterolaparoscopy with normal husband semen analysis as a part of their infertility workup at M. G. M. Medical Hospital, Kalamboli. Sample size: 30.

Results: Incidence of GTB=22.85\%, 25-29 year followed by 35-40 years' age group was the most common age group was observed. In our study, $74.3 \%$ infertility pattern was primary, $25.7 \%$ were secondary only $2.85 \%(\mathrm{n}=1)$ of the cases of GTB were diagnosed by using TB BACTEC. Laparoscopic findings su0ggested that 18 cases had normal findings and 8 cases had laparoscopic features suggestive of GTB. On chromo pertubation, delayed and absent spillage of the dye was seen in 2 cases. On Hysteroscopy, 29 cases (82.9\%) of the patients had normal findings and 3 cases had Hysteroscopic features suggestive of GTB.

Conclusions: Genital tuberculosis remains an important under diagnosed cause of infertility. Though culture is considered as gold standard for diagnosis of genital tuberculosis, since GTB is paucibacillary there is an urgent need for more research to come to conclusion whether culture is still gold standard. Further research is required to detect the most sensitive method for diagnosis.

Keywords: Bactec culture, Genital tuberculosis, Hysterolaparoscopy, Infertility

\section{INTRODUCTION}

Infertility is agonising condition and trauma of infertility is better felt and described by the infertile couple themselves. Infertility (subfertility) is defined as one year of unprotected intercourse without conception. Causes of female infertility are ovulatory dysfunction (20-40\%), tubal and peritoneal pathology (30-40\%), uterine pathology (uncommon) and unexplained. Overall fertility rates are 4-8\% lower in women aged 25-29 years, $15-19 \%$ lower in aged 30-34\%, 26-46\% lower in women aged 3539 years, 95\% lower for women aged 40-45 years. Laparoscopy and Hysterosalpingogram are the two classic methods for evaluation of tubal and uterine factors. Laparoscopy is an important tool to assess the reproductive pathology including tubal patency (chromopertubation) in infertile women. It provides both panoramic view of pelvic anatomy and a magnified view of uterine, ovarian, tubal, and peritoneal surfaces and its pathology. Tuberculosis is an important health problem worldwide. One third of the world's population is currently affected with tuberculosis.

In India, every year 1.8 million people develop the disease, of which 80,000 are infectious, 1000 die of it every day, with two deaths occurring every three minutes. Genital tuberculosis represents $15-20 \%$ of extra pulmonary 
tuberculosis and is the second most common site infected after pulmonary tuberculosis. Genital tuberculosis is seen in $12 \%$ of all patients with pulmonary tuberculosis. It accounts for $5-10 \%$ of all pelvic infections.

\section{Robert heinrich herman koch}

He was a German physician and the founder of modern bacteriology. He won Nobel Prize for his research contribution in the year 1905. Robert Koch was also known for his role in identifying the specific causative agent of tuberculosis, cholera, anthrax. ROBERT KOCH research led to the creation of Koch's postulates, a series of 4 generalized principles linking specific microorganism to specify disease that remain today the 'Gold Standard' in medical microbiology.

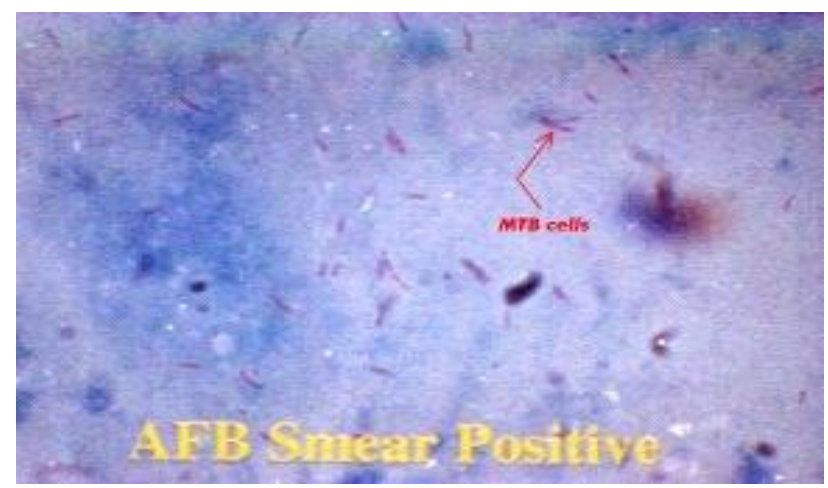

Figure 1: Mycobacterium tuberculous slide.

Tuberculosis is an important and commonest health problem worldwide. One third of the population is infected with tuberculosis. A single infected person is known to infect 10 or more people per year. Genital tuberculosis is the second most common site infected after pulmonary tuberculosis.

\section{Microscopic appearance}

Typical granuloma consists of infiltration of multinucleated giant cells (LANGHANS'), chronic inflammatory cells, epithelioid cells, surrounding a central area of caseation necrosis.

Study by Sutherland and Macfarlane, 128 husbands of women with genital tuberculosis only $5(3.9 \%)$ were found to have active genital tuberculosis (TELINDE'S). The introduction of broth based growth systems, has significantly reduced the time to detection and increased the total number of positive cultures. The first such system developed was the BACTECMGIT 960 system (Mycobacterium growth indicator tube) system was produced by Becton Dickinson Cockins Ville, Mary Land USA. MGIT contains $7 \mathrm{ml}$ of modified middle brook $7 \mathrm{~h} 9$ broth base. The complete medium with OADC enrichment and PANTA antibiotic mixture most commonly used liquid media for cultivation of mycobacterium. BACTEC uses palmitic acid as the substrate for the growth of AFB.
The carbon atoms in this substrate are radio-labelled and thus the carbon dioxide $(\mathrm{CO} 2)$ released is also radiolabelled and this is measured in the form of growth index. A growth index of more than 10 gives a strong suspicion of TB. BACTEC gives rapid results and is highly sensitive and the time needed for culture and drug sensitivity testing is about 2 to 3 wks. Colonies are seen even if the bacillary count is $100 .{ }^{1,2}$ BACTEC has a sensitivity of $80-90 \%$ whereas $\mathrm{L} \mathrm{J}$ medium has sensitivity as 30-40\%. Tuberculosis is an age-old disease but is amongst the foremost killers of the $21^{\text {st }}$ century.

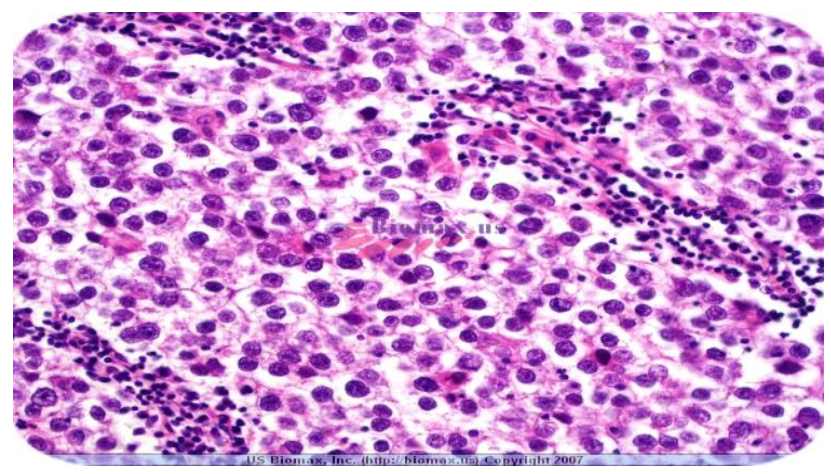

Figure 2: Genital tuberculosis slide.

The highest rates of TB are in some of the world's poorest countries, and the economic toll taken by the disease is enormous. It is a major health problem in India and is responsible for a significant proportion of women presenting with infertility. The actual incidence of genital tuberculosis cannot be assessed accurately, since the disease is discovered incidentally in many patients and in a large number of asymptomatic patients this disease remains undiscovered. It is estimated that $5-10 \%$ of infertile women all over the world have genital tuberculosis although this varies from less than $1 \%$ in the United States to nearly $18 \%$ in India. It is found in 0.75 to $1 \%$ of all Gynaecological admissions in India. ${ }^{3,4}$ The disease is responsible for $5 \%$ of all female pelvic infections and occurs in $13 \%$ cases of pulmonary tuberculosis.3Although genital tuberculosis can occur in any age group, $75 \%$ of the patients are in the reproductive age group (25-45 years). Postmenopausal women account for $7-11 \%$ cases of GTB. ${ }^{4}$

Objectives of the study to correlate the hysterolaparoscopic and bactec culture in infertility.

\section{METHODS}

\section{Source of data}

Infertile women undergoing dilatation and curettage with hysterolaparoscopy with normal husband semen analysis as a part of their infertility workup at M. G. M. Medical Hospital, Kalamboli. Sample size is 30 , Setting of study in M.G.M Medical Hospital Kalamboli Navi Mumbai.

\section{Inclusion criteria}


Infertile women subjected to dilatation and curettage with hysterolaparoscopy with normal husband semen analysis as a part of infertility workup after counselling the patient

Diagnostic laparoscopy with 7mm Karl Storz laparoscope with 30-degree deflection angle powered with fibre-optic table and light source with dilatation and curettage will be performed in the pre-menstrual period between days 18-22 of the cycle for infertility evaluation. A careful evaluation of the fallopian tubes, ovaries, pelvic peritoneum, pouch of Douglas and peritoneal cavity will be done.

\section{Features suggestive of genital tuberculosis will be looked for by noting the presence}

Miliary tubercles on the uterus and tubes, Nodular salpingitis, Caseosalpinx, Hydrosalpinx, Presence of peritubal, periovarian, omental and bowel adhesions, Free fluid in the pouch of Douglas.

Following this hysteroscopy using normal saline as the distention media was done to identify features suggestive of tuberculosis that included:

\section{Presence of tubercles \\ 2. Microcaseation \\ 3. Distorted ostia \\ 4. Calcifications \\ 5. Synechiae}
a) Grade 1 - less than 1/4th of uterine cavity involved
b) Grade 2 - 1/4th to 3/4th of uterine cavity involved; ostia and fundus partly involved
c) Grade 3 - more than 3/4th of uterine cavity involved; ostia and upper cavity occluded.

Chromopertubation was done and any delayed or absent spillage of dye was noted. At the end of the procedure endometrial curettings were taken and sent for BACTEC in normal saline. Video recording of all the cases was done. In cases found to be positive for genital tuberculosis by BACTEC of endometrial curetting's the clinical findings were analyzed and video recordings of the hysterolaparoscopic findings were carefully evaluated for the presence of features suggestive of genital tuberculosis.

\section{RESULTS}

In the Table 1 it was observed that most of the study population was between 25-29 years of age group followed by $35-40$ years.

Table 1: Age distribution.

\begin{tabular}{|lll|}
\hline Age group & N & $\%$ \\
\hline $15-19$ & 1 & $2.8 \%$ \\
\hline $20-24$ & 2 & $5.7 \%$ \\
\hline $25-29$ & 16 & $45.71 \%$ \\
\hline $30-34$ & 7 & $20 \%$ \\
\hline
\end{tabular}

\begin{tabular}{|lll|}
\hline $35-40$ & 9 & $25.71 \%$ \\
\hline Grand Total & $\mathbf{3 5}$ & $\mathbf{1 0 0 \%}$ \\
\hline
\end{tabular}

Table 2: Infertility pattern.

\begin{tabular}{|lll|}
\hline & Frequency & Percent \\
\hline Primary & 26 & 74.3 \\
\hline Secondary & 9 & 25.7 \\
\hline Total & 35 & 100.0 \\
\hline
\end{tabular}

Table 2 showing $74.3 \%$ infertility pattern was primary and $25.7 \%$ were secondary.

Table 3: Different menstrual patterns.

\begin{tabular}{|lll|}
\hline & Frequency & Percent \\
\hline Hypomenorrhoea & 2 & 5.7 \\
\hline Normal & 25 & 71.4 \\
\hline Menorragia & 2 & 5.7 \\
\hline Oligomenorrhoea & 6 & 17.1 \\
\hline Total & 35 & 100.0 \\
\hline
\end{tabular}

Majority of the women had normal menstrual function (71.4\%), Oligomenorrhea (17.1), Hypomenorrhea (5.7), Amenorrhea (2.9).

Table 4: BACTEC findings.

\begin{tabular}{|l|l|}
\hline $\begin{array}{l}\text { Endometrial curettings } \\
\mathrm{n}=35\end{array}$ & $1(2.85 \%)$ \\
\hline
\end{tabular}

Table 5: Laparoscopic findings.

\begin{tabular}{|l|l|l|}
\hline Laparoscopy findings & No. of cases & Percentage \\
\hline Normal Cases & 18 & 51.42 \\
\hline $\begin{array}{l}\text { Peritubal/Periovarian } \\
\text { Adhesions }\end{array}$ & 5 & 14.28 \\
\hline Hydrosalpinx & 2 & 5.71 \\
\hline Fluid in POD & 1 & 2.8 \\
\hline
\end{tabular}

All the patients who underwent hysterolaparoscopy had endometrial curettings sent for TB BACTEC was positive in $2.85 \% \quad(n=1)$, and none of the cases had histopathological evidence of genital TB. On laparoscopy, 18 cases $(51.42 \%)$ of the patient had normal findings and 8 cases had laparoscopic features suggestive of GTB. Peritubal or Periovarian Adhesions, Hydrosalpinx, Fluid in POD was present in 5,2,1 cases respectively. On Hysteroscopy, 29 cases $(82.9 \%)$ of the patients had normal findings and 3 cases had Hysteroscopic features suggestive of GTB. Bald endometrium, Distorted ostium, Synechiae was present in 1,1,1 cases respectively.

Table 6: Hysteroscopy findings.

\begin{tabular}{|lll|}
\hline Hysteroscopy & Frequency & Percent \\
\hline Bald endometrium & 1 & 2.9 \\
\hline Distorted ostium & 1 & 2.9 \\
\hline
\end{tabular}




\begin{tabular}{|lll|} 
Normal study & 29 & 82.9 \\
Synechiae & 1 & 2.9 \\
\hline
\end{tabular}

\section{DISCUSSION}

Female genital tuberculosis affects the females of reproductive age group. The disease manifests itself as pelvic inflammatory disease in its acute form with menstrual irregularities and later infertility, and is almost always secondary to a primary lesion elsewhere. The disease frequently remains unnoticed even in most of the tertiary health care set ups particularly in developing countries. The true incidence of this is important but relatively ignored form of extra pulmonary tuberculosis remains unknown 5 as the disease possess unconquered diagnostic challenges mainly because the primary symptoms are usually non-characteristic.

Infertility is a well-known as sequelae. Early diagnosis invariably helps to speed up the decision-making process and markedly reduces the time lag in starting antitubercular therapy. As a result, the disease is either not diagnosed or diagnosed at an advanced stage when the tubal damage has already occurred. At this stage, the prognosis for fertility is poor despite treatment.

Current methods of diagnosis of genital tuberculosis in most set ups largely rely on clinical parameters combined with endoscopic findings and the antiquated conventional microbiological tests. With regard to incidence of genital tuberculosis, it is often difficult to gauge the actual magnitude of the problem as the disease initially presents without symptoms and due to diagnostic difficulties, it is picked only during the investigation for infertility. The incidence also varies greatly according to the socioeconomic and public health conditions; therefore, there is wide variation in figures published from various countries. ${ }^{6,7}$

In the present study, criteria for diagnosis of GTB was positive TB BACTEC and granulomatous lesion on histopathology. Hysterolaparoscopy findings in the cases found to be positive for GTB were carefully reviewed for structural lesions suggestive of GTB. Several other studies have quoted the incidence of GTB using combined diagnostic tests including hysteroscopy and laparoscopy findings which may be quite nonspecific. ${ }^{8}$

In the present study, the incidence of genital tuberculosis was $22.85 \%$. A study done in South Africa on 109 infertile women where the diagnosis of genital tuberculosis was made using positive culture of AFB from three samples of endometrial tissue, menstrual blood and peritoneal fluid from pouch of Douglas, the incidence of GTB was found to be $21 \%$.

In the present study, it was observed that 25 -29 years of age group followed by 35-40 years was the most common age group amongst study population. The mean age of the study population $29.66 \pm 4.72$ years. In the study conducted by Bapna Neelam et al., 2005 the median age of women in our study was 31 years (range 22-40 years). ${ }^{10}$

In the present study, $74.3 \%$ infertility pattern was primary and $25.7 \%$ were secondary. Majority of the women had normal menstrual function (71.4\%), Oligomenorrhea (17.1), Hypomenorrhea (5.7), Amenorrhea (2.9) Similar findings were reported in an Indian study where normal menstrual pattern was seen in $57.6 \%$ of cases and hypomenorrhoeain $30.1 \%$ cases. $^{11}$

In our study, only $2.85 \%(n=1)$ of the cases of GTB were diagnosed by using TB BACTEC (culture). A low pick up rate in our study is probably due to the fact that endometrial biopsy was done in the postmenstrual phase. ${ }^{12}$

Laparoscopy is an important tool in the evaluation of infertility and in the diagnosis of various pelvic conditions. In women with high suspicion of genital tuberculosis, endoscopy helps to obtain microbiological samples under direct vision, evaluate the extent of damage and treat the patient accordingly.

In the present study, laparoscopic findings suggested that 18 cases $(51.42 \%)$ of the study patients had normal findings and 8 cases had laparoscopic features suggestive of GTB. Peritubal or Periovarian Adhesions, Hydrosalpinx, Fluid in POD was present in $62.50 \%$, $25 \%, 12.5 \%$ cases respectively. In the study conducted by Baxi A et al., 2011, Peritubal or Periovarian Adhesions was present in $20.11 \%$ cases. $^{13}$

On chromo pertubation, delayed and absent spillage of the dye was seen in 2 cases which is correlating well with the figures obtained from other studies which have reported abnormal chromopertubation results in $50 \%$ cases. This is due to the fact that fallopian tubes are the initial and most frequently affected site in pelvic mycobacterial infection. Fluid in pouch of Douglas was detected in $12.5 \%$ cases while other authors have reported in $8.45 \%$ cases. Normal laparoscopic findings were observed in $51.42 \%$ cases which could be due to the fact that it generally detects macroscopic Changes that are seen in chronic stages. ${ }^{14}$

On Hysteroscopy, 29 cases $(82.9 \%)$ of the patients had normal findings and 3 cases had Hysteroscopic features suggestive of GTB. Bald endometrium, Distorted ostium, Synechiae was present in $12.5 \%, 12.5 \%, 12.5 \%$ cases respectively. In the study conducted by Asha baxi et al., 2011 the Intrauterine synechie was observed only in $3.57 \%$ and fibrosed ostia in $7.14 \% .^{13}$

\section{CONCLUSION}

Genital tuberculosis remains an important under diagnosed cause of infertility. The alarming high incidence of genital TB underlines the importance of investigating and treating this condition in all infertile women. Though culture is considered as gold standard for diagnosis of genital tuberculosis, since GTB is paucibacillary there is an urgent 
need for more research to come to conclusion whether culture is still gold standard. Further research is required to detect the most sensitive method for diagnosis. GTB and therefore the diagnostic dilemma continues.

Funding: No funding sources

Conflict of interest: None declared

Ethical approval: The study was approved by the Institutional Ethics Committee

\section{REFERENCES}

1. Jassawalla MJ. Genital Tuberculosis- A Diagnostic Dilemma. J ObstetGynecol India. 2006;56(3):203-4.

2. Lakshmi V, Patil MA, Subhadha K, Himabindu V. Isolation of Mycobacteria by BACTEC 460 system from clinical specimens. Ind. J Med Micribiology, 2006;24(2):124-6.

3. Arora VK, Gupta R, Arora R. Female Genital Tuberculosis: Need for More Research. Ind J Tub. 2003;50(9):36-8.

4. Gatongi DK, Gitau G, Kay V, Ngwenya S, Lafong C, Hasan A. Female genital tuberculosis. ObstetGynaecol. 2005;7:75-9.

5. Litynski GS. Laparoscopy. The early attempts: spotlighting Georg Kelling and Hans Christian Jacobaeus. Journal of the Society of Laparoendoscopic Surgeons. 1997;1:83-5.

6. Vecchio R, MacFayden BV, Palazzo F. History of laparoscopic surgery. Panminerva Med. 2000;42(1):87-90.

7. Advincula AP, Wang K. Evolving role and current state of robotics in minimally invasive gynecologic surgery. J Minim Invasive Gynecol. 2009;16(3):291301.

8. Rowe PJ, Comhaire FH, Hargreave TB, Mahmoud AMA. WHO manual for the standardized investigation of the infertile couple. Cambridge, UK: Cambridge University Press Cambridge, UK, 1993.

9. Forman RG, Robinson JN, Mehta Z, Barlow DH. Patient history as a simple predictor of pelvic pathology in sub fertile women. Hum Reprod.1993;8:53-5.

10. Balasch J. Investigation of the infertile couple in the era of assisted reproductive technology: A time for reappraisal. Human Reproduction 2000; 15: 2251-57.

11. Simon A, Laufer N. Unexplained infertility: a reappraisal. Ass Reprod Rev. 1993;3:26-36.

12. Fayez JA, Mutie G, Schneider PJ. The diagnostic value of hysterosalpingography and laparoscopy in infertility investigation. Int J Fertil. 1988;33:98-101.

13. Comparative studies between the value of hysterosalpingography and Coelioskopie in sterility diagnosis. Archives of Gynecology and Obstetrics. 1967:204;167-8.

14. Marc JNC Keirse, Vandervellen R. A comparison of HSG and Laparoscopy in the investigation of infertility. Obstetrics and Gynecology. 1973;41(5):685-8

Cite this article as: Guru M, Deshmukh PY. A correlation of hysterolaparoscopic findings and bactec culture in infertility. Int J Reprod Contracept Obstet Gynecol 2017;6:1430-4. 